\title{
Complete Inference Systems for Weak Bisimulation Equivalences in the $\pi$-Calculus
}

\author{
Huimin Lin* \\ Institute of Software, Chinese Academy of Sciences \\ P.O.Box 8718, Beijing 100080 \\ E-mail: lhm@ios.ac.cn
}

\begin{abstract}
Proof systems for weak bisimulation equivalences in the $\pi$-calculus are presented, and their soundness and completeness are shown. The proofs of the completeness results rely on the notion of symbolic bisimulation. Two versions of $\pi$-calculus are investigated, one without and the other with the mismatch construction. For each version of the calculus proof systems for both late and early weak bisimulation equivalences are studied. Thus there are four proof systems in all. These proof systems are related in a natural way: the proof systems for early and late equivalences differ only in the inference rule for the input prefix, while the proof system for the version of $\pi$-calculus with mismatch is obtained by adding a single inference rule for the version without it.
\end{abstract}

\section{Introduction}

This paper aims to provide complete proof systems for weak bisimulation equivalences in the $\pi$-calculus. Bisimulation equivalences are among the most welldeveloped semantic theories for process calculi. The $\pi$-calculus inherits from $C C S$ the invisible $\tau$ action, so the usual distinction between strong and weak bisimulations applies: the strong version gives the $r$ action the same weight as any other normal, or visible, actions, while the weak version ignores $\tau$ moves. As $\tau$ action is used to represent internal communication and as such is not observable, the weak equivalence is what used for practical applications. On the other hand, for message-passing process calculi, of which the $\pi$-calculus is a special instance, another distinction can be made, namely late and early bisimulations [MPW92, HL92], according to when input variables are instantiated. Thus for the $\pi$-calculus we have at least four bisimulation equivalences to study.

The original $\pi$-calculus paper [MPW92] only gave an axiomatisation for the late version of strong ground bisimulation. Subsequently, efforts have been made to formulate complete proof systems for other equivalences for this calculus: [PS93, BD94] for both early and late strong bisimulation congruences,

*This work has been supported by research grants from the President Fund of the Chinese Academy of Sciences and the Natural Science Foundation of China 
[Hen91, BD92] for testing equivalence, and [San93] for (strong) open bisimulation. Axiomatisations for weak bisimulations are still missing, though it has been widely conjectured that they can be obtained by adding Milner's $\tau$-laws to proof systems for strong equivalences.

In [Lin94b] we presented proof systems for strong bisimulation equivalences in the $\pi$-calculus. These proof systems consist of a set of inference rules together with some standard equations. The judgements of the inference systems are of the form

$$
C \triangleright t=u
$$

where $t, u$ are $\pi$-calculus terms and $C$, called a condition, is a set of equality or inequality tests between names. It is important to note that $C$ is not a construction in the $\pi$-calculus; it is a construction used in our meta language in order to reason about bisimulation equivalences for the calculus. To give a taste of the proof system, here is the inference rule for match:

$$
\text { MATCH } \frac{C \cup\{x=y\} \triangleright t=u \quad C \cup\{x \neq y\} \triangleright \mathbf{0}=u}{C \triangleright[x=y] t=u}
$$

It involves a case analysis: if we can establish $t=u$ under the condition $x=y$, and $\mathbf{0}=u$ under the condition $x \neq y$, then we can conclude $[x=y] t=u$. Here it can be seen clearly how the inequalities in the meta-language help to characterise constructions in the calculus. For the extension of the $\pi$-calculus with the mismatch operator, only a single inference rule, which is dual to the above rule for match, has to be added to the proof systems.

In this paper we shall extend these results to weak bisimulation equivalences. It turns out that, as in CCS, the three $\tau$-laws ([Mil89]) are sufficient for this purpose. We shall demonstrate how, by exploiting the notion of maximally consistent extensions of a condition, the standard techniques used to prove the completeness results in $C C S$ can be re-cast for the $\pi$-calculus.

As in CCS, weak bisimulations in the $\pi$-calculus are defined in terms of double arrows that abstract away $\tau$-moves. But care must be taken in dealing with late equivalence: in this case input moves should not be allowed to absorb the $\tau$ moves after them, for otherwise the resulted relation would not be transitive. Also as in $C C S$, weak bisimulations are not preserved by the choice operator, so we have to work with observation equivalences.

The proofs of the completeness results for our inference systems rely on the notion of symbolic bisimulations [HL92, HL93]. In [MPW92] a general notion of bisimulation, $\sim^{D}$, called distinction indexed bisimulation, is introduced, where the index $D$ is a set of inequations on names such that $t \sim^{D} u$ iff $t \sigma \dot{\sim} u \sigma$ for every substitution $\sigma$ that satisfies $D$. In the setting of the $\pi$-calculus, symbolic bisimulation is a mild generalisation of distinction indexed bisimulation, it is indexed by conditions consisting of name inequations as well as name equations. This generalisation makes it possible to give a direct definition of symbolic bisimulation (in terms of symbolic transitional semantics) instead of as substitution closure on top of ground bisimulation, which facilitates the proofs of the completeness results for the inference systems. 
The main advantage of working with $\pi$-calculus over general message-passing calculi is that the language for the message domain is known: it is simply a set of port names upon which the only allowed operations are tests for equality and inequality. To define the notion of symbolic bisimulation for the $\pi$-calculus, we first develop a simple theory of conditions which are sets of equalities and inequalities over names. A maximally consistent condition is a complete, or saturated, condition, in the sense that adding to it anything not implied by it will result in inconsistency. This notion plays an important rôle in the definition of symbolic bisimulation and in the proofs of the completeness results.

The rest of this paper is organised as follows: The calculus and its semantics are introduced in the next section. The inference system is presented in Section 3, along with the completeness proof. Section 4 demonstrates how the theory developed for the late equivalence in the previous sections can be carried over to the early case. Section 5 discusses extensions to the calculus. The paper is concluded with Section 6, where related work is also discussed.

Due to space limitation, most of the proofs have been omitted. They can be found in the full version of this paper [Lin94a].

\section{The $\pi$-Calculus And Bisimulations}

\subsection{Names and Conditions}

The basic entities of the $\pi$-calculus are names, i.e. identifiers for communication channels. Let $\mathcal{N}$, ranged over by $x, y, z, \ldots$ be a countably infinite set of names.

Conditions, ranged over by $C, D$, are finite sets of equality or inequality tests on names. We will write $n(C)$ for the set of names appearing in $C$. We say $C$ is a condition on a name set $V$ if $n(C) \subseteq V$. The empty condition will sometimes be denoted by true. Matches, ranged over by $M, N, L$, are special conditions which consist of only name equalities. Matches are part of the $\pi$-calculus, while conditions are only used in our meta-theory about the $\pi$-calculus, namely in the definition of symbolic bisimulations and in the formulation of inference rules.

Substitutions, ranged over by $\sigma$ and $\delta$, are mappings from $\mathcal{N}$ to $\mathcal{N}$, and $[\bar{y} / \bar{x}]$ is the substitution sending $\bar{x}$ to $\bar{y}$ and is identity otherwise. Substitutions are postfix operators, and have higher precedence than the operators in the language. If $\sigma=[\bar{y} / \bar{x}]$ then $n(\sigma)=\{\bar{y}\} \cup\{\bar{x}\}$. So for any $x \notin n(\sigma), y \sigma=x$ iff $y=x$.

A substitution $\sigma$ satisfies a condition $C$, written $\sigma \models C$, if $x \sigma=y \sigma$ for any $x=y \in C$ and $x \sigma \neq y \sigma$ for any $x \neq y \in C$. We write $C \Rightarrow D$ to mean that $\sigma \models C$ implies $\sigma \models D$ for any substitution $\sigma$.

The elements in a condition are treated as conjuncts. We avoid introducing disjunction into the theory of conditions. The major advantage is that the relation $C \Rightarrow D$ can be tested in linear time w.r.t the size of $C$ and $D$ :

Proposition 2.1 The relation $C \Rightarrow D$ is linear time decidable.

Allowing disjunction does not affect our theoretical results. The only consequence is that the above implication relation would in general take exponential time to decide. 
Two substitutions $\sigma$ and $\sigma^{\prime}$ are equal on $V$, written $\sigma={ }^{V} \sigma^{\prime}$, if $x \sigma=x \sigma^{\prime}$ for all $x \in V$. A condition $C$ is consistent if there are no $x, y \in \mathcal{N}$ such that $C \Rightarrow x=y$ and $C \Rightarrow x \neq y . C$ is maximally consistent on $V \subset \mathcal{N}$ if for any $x, y \in V$ either $C \Rightarrow x=y$ or $C \Rightarrow x \neq y$.

$C^{\prime}$ is a maximally consistent extension of $C$ on $V$, written $C^{\prime} \in M C E_{V}(C)$, if $C \subseteq C^{\prime}$ and $C^{\prime}$ is maximally consistent on $V$. The set of maximally consistent extensions of a given condition on a finite set of names $V$ is finite. We will abbreviate $M C E_{V}($ true $)$ as $M C_{V}$.

Lemma 2.2 $\vee M C E_{V}(C)=C$

Lemma 2.2 shows that the set of all consistent extensions of a condition (on a given name set) constitutes a boolean partition ([HL92, HL93]) of the condition.

Proposition 2.3 Suppose $C$ is maximally consistent on $V$. If $\sigma$ and $\sigma^{\prime}$ both satisfy $C$, then $\sigma={ }^{V} \sigma^{\prime} \delta$ for some injective substitution $\delta$.

\subsection{The Language And Operational Semantics}

The language of $\pi$-calculus can be given by the following BNF grammar

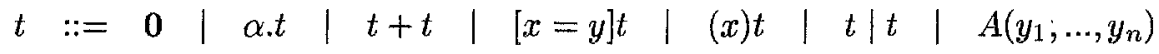

$$
\begin{aligned}
& \alpha::=\tau|a(x)| \bar{a} x
\end{aligned}
$$

We refer to [MPW92] for intended meanings of these operators.

In $a(x) . t$ and $(x) t x$ is a bound name with scope $t$. We use $b n(t)$ and $f n(t)$ for the set of bound and free names in $t$, respectively. Bound names induce the notion of $\alpha$-equivalence as usual. In the sequel we will not distinguish between $\alpha$-equivalent terms, using $\equiv$ for both syntactical equality and $\alpha$-equivalence. In the $\pi$-calculus no clear distinction is made between constants and variables, and we will call "processes" those terms whose free names are not to be instantiated.

The operational semantics of the language is reported in Figure 1, where a transition is of the form $t \stackrel{\alpha}{\rightarrow} u$ with $\alpha$ ranging over four kinds of actions: $\tau, a(x), \bar{a} x$ and $\bar{a}(x)$. We have omitted the symmetric rules for sum and par. Transitions are defined upto $\alpha$-equivalence, i.e. $\alpha$-equivalent terms are deemed to have the same transitions. Bound names for actions are defined by: $b n(a(x))=b n(\bar{a}(x))=\{x\}$ and $b n(\tau)=b n(\bar{a} x)=\emptyset$.

We write $\alpha={ }^{C} \beta$ to mean

$$
\begin{aligned}
& \text { if } \alpha \equiv \tau \text { then } \beta \equiv \tau \\
& \text { if } \alpha \equiv \bar{a} x \text { then } \beta \equiv \bar{b} y \text { and } C \Rightarrow a=b, C \Rightarrow x=y \\
& \text { if } \alpha \equiv \bar{a}(x) \text { then } \beta \equiv \bar{b}(x) \text { and } C \Rightarrow a=b \\
& \text { if } \alpha \equiv a(x) \text { then } \beta \equiv b(x) \text { and } C \Rightarrow a=b
\end{aligned}
$$

The transitions defined in Figure 1 are concrete in the sense that they will always be fired regardless the contexts in which terms are placed. In our work 


$$
\begin{aligned}
& \text { pre } \frac{}{\alpha . t \stackrel{\alpha}{\longrightarrow} t} \\
& \operatorname{sum} \frac{t \stackrel{\alpha}{\rightarrow} t^{\prime}}{t+u \stackrel{\alpha}{\rightarrow} t^{\prime}} \quad \operatorname{par} \frac{t \stackrel{\alpha}{\longrightarrow} t^{\prime}}{t\left|u \stackrel{\alpha}{\longrightarrow} t^{\prime}\right| u} b n(\alpha) \cap f n(u)=\emptyset \\
& \operatorname{res} \frac{t \stackrel{\alpha}{\longrightarrow} t^{\prime}}{(x) t \stackrel{\alpha}{\longrightarrow}(x) t^{\prime}} x \notin n(\alpha) \quad \operatorname{com} \frac{t \stackrel{a(x)}{\longrightarrow} t^{\prime} u \stackrel{\bar{a} y}{\longrightarrow} u^{\prime}}{t\left|u \stackrel{\tau}{\longrightarrow} t^{\prime}[y / x]\right| u^{\prime}} \\
& \text { open } \frac{t \stackrel{\bar{a} x}{\longrightarrow} t^{\prime}}{(x) t \stackrel{\bar{a}(x)}{\longrightarrow} t^{\prime}} a \neq x \quad \text { close } \frac{t \stackrel{a(x)}{\rightarrow} t^{\prime} u \stackrel{\bar{a}(x)}{\longrightarrow} u^{\prime}}{t \mid u \stackrel{\tau}{\longrightarrow}(x)\left(t^{\prime} \mid u^{\prime}\right)} \\
& \text { id } \frac{t\left[y_{1}, \ldots, y_{n} / x_{1}, \ldots, x_{n}\right] \stackrel{\alpha}{\rightarrow} t^{\prime}}{A\left(y_{1}, \ldots, y_{n}\right) \stackrel{\alpha}{\longrightarrow} t^{\prime}} A\left(x_{1}, \ldots, x_{n}\right) \stackrel{\text { def }}{=} t
\end{aligned}
$$

Figure 1: $\pi$-Calculus Transitional Semantics

on general message-passing processes [HL92, HL93] we use a more abstract form of transitions called symbolic transitions. A symbolic transition takes the form $t \stackrel{b, \alpha}{\longrightarrow} u$, where $b$ is a boolean condition. Intuitively $b$ represents the context under which action $\alpha$ can actually be fired from $t$. In the setting of the $\pi$-calculus $b$ will be a set of matches, i.e. equality tests on names. This kind of transition has also been used in the work of open bisimulation by Sangiorgi [San93].

The symbolic transitional semantics of the $\pi$-calculus is given in Figure 2. For notational convenience we write $M N$ for the union of $M$ and $N$. Again the symmetric rules for Sum and Par have been omitted.

\subsection{Weak Bisimulations}

Strong bisimulations for the $\pi$-calculus and their proof systems have been studied in [Lin94b], so here we only consider weak equivalences. For the bulk of this paper we will concentrate on late bisimulation, and we will sketch in a later section how these results can be carried over to early bisimulation in a systematic manner. So until Section 4, the word "bisimulation" means "late bisimulation".

As in CCS, weak bisimulation is defined in terms of double arrows which ignore $\tau$-moves. But late bisimulation insists that an input move from one process be matched by a single input move from the other. As a consequence, in defining late double arrows input moves should not be allowed to absorb $\tau$ moves after them.

The (concrete) late double arrow relation is generated by the following rules

- $p \stackrel{\varepsilon}{=} l p$.

- $p \stackrel{\alpha}{\longrightarrow} l q$ implies $p \stackrel{\alpha}{\Longrightarrow} q$. 


$$
\begin{aligned}
& \text { Pre } \frac{t \stackrel{M, \alpha}{\longrightarrow} t^{\prime}}{\alpha \cdot t^{\text {true } \alpha} \rightarrow} t \quad \text { Match } \frac{t}{[x=y] \stackrel{M L, \alpha}{\longrightarrow} t^{\prime}} \quad L= \begin{cases}x=y & \text { if } x \neq y \\
\emptyset & \text { otherwise }\end{cases} \\
& \operatorname{Sum} \frac{t \stackrel{M, \alpha}{\longrightarrow} t^{\prime}}{t+u \stackrel{M, \alpha}{\longrightarrow} t^{\prime}} \quad \operatorname{Par} \frac{t \stackrel{M, \alpha}{\longrightarrow} t^{\prime}}{t\left|u \stackrel{M, \alpha}{\longrightarrow} t^{\prime}\right| u} b n(\alpha) \cap f n(u)=\emptyset \\
& \operatorname{Res} \frac{t \stackrel{M, \alpha}{\rightarrow} t^{\prime}}{(x) t \stackrel{M, \alpha}{\rightarrow}(x) t^{\prime}} x \notin n(M, \alpha) \quad \text { Open } \frac{t \stackrel{M, \bar{a} x}{\longrightarrow} t^{\prime}}{(x) t \stackrel{M, \bar{a}(x)}{\longrightarrow} t^{\prime}} x \notin n(M, a) \\
& \operatorname{Com} \frac{t \stackrel{M, a(x)}{\longrightarrow} t^{\prime} u \stackrel{N, \bar{b} y}{\longrightarrow} u^{\prime}}{t\left|u \stackrel{M N L, \tau}{\longrightarrow} t^{\prime}[y / x]\right| u^{\prime}} L= \begin{cases}a=b & \text { if } a \neq b \\
\emptyset & \text { otherwise }\end{cases} \\
& \text { Close } \frac{t \stackrel{M, a(x)}{\longrightarrow} t^{\prime} u \stackrel{N, \bar{b}(x)}{\longrightarrow} u^{\prime}}{t \mid u \stackrel{M N L, \tau}{\longrightarrow}(x)\left(t^{\prime} \mid u^{\prime}\right)} \quad L= \begin{cases}a=b & \text { if } a \neq b \\
\emptyset & \text { atherwise }\end{cases} \\
& \text { Id } \frac{t\left[y_{1}, \ldots, y_{n} / x_{1}, \ldots, x_{n}\right] \stackrel{M, \alpha}{\longrightarrow} t^{\prime}}{A\left(y_{1}, \ldots, y_{n}\right) \stackrel{M, \alpha}{\longrightarrow} t^{\prime}} A\left(x_{1}, \ldots, x_{n}\right) \stackrel{\text { def }}{=} t
\end{aligned}
$$

Figure 2: $\pi$-Calculus Symbolic Transitional Semantics

- $p \stackrel{\tau}{\longrightarrow} \stackrel{\alpha}{\Longrightarrow}_{l} q$ implies $p \stackrel{\alpha}{\Longrightarrow} q$.

- If $\alpha$ does not have the form $a(x)$ then $p \stackrel{\alpha}{\Longrightarrow} \stackrel{\tau}{\longrightarrow}_{l} q$ implies $p \stackrel{\alpha}{\Longrightarrow} q$.

Let $\stackrel{\hat{\jmath}_{\rightleftharpoons}}{\Longrightarrow}$ be $\stackrel{\varepsilon}{\Longrightarrow} l$, and $\stackrel{\hat{\alpha}}{\Longrightarrow}$ be $\stackrel{\alpha}{\Longrightarrow}$ for $\alpha \not \equiv \tau$.

Definition 2.4 A symmetric relation $R$ is a late weak ground bisimulation if $(p, q) \in R$ implies:

- whenever $p \stackrel{a(x)}{\longrightarrow} p^{\prime}$ with $x \notin f n(p, q)$ then $q \stackrel{a(x)}{\longrightarrow} l q^{\prime \prime}$ for some $q^{\prime \prime}$ s.t for any $y$ there is $q^{\prime \prime}[y / x] \stackrel{\hat{\tau}}{\Longrightarrow} l q^{\prime}$ and $\left(p^{\prime}[y / x], q^{\prime}\right) \in R$.

- whenever $p \stackrel{\alpha}{\longrightarrow} p^{\prime}$ for any other action $\alpha$ with $b n(\alpha) \cap f n(p, q)=\emptyset$ then $q \stackrel{\hat{\alpha}}{\Longrightarrow} l q^{\prime}$ for some $q^{\prime}$ and $\left(p^{\prime}, q^{\prime}\right) \in R$.

Write $p \dot{\approx}_{l} q$ if there exists a late weak ground bisimulation $R$ s.t. $(p, q) \in R$. $t$ is late weak bisimular to $u$, written $t \approx_{l} u$, if $t \sigma \dot{\approx}_{l} u \sigma$ for every substitution $\sigma$.

Similar to $C C S, \approx_{l}$ is not preserved by + . So we have to work with the modified relation:

Definition $2.5 p$ and $q$ are late ground observation equivalent, written $p \dot{\check{\simeq}}_{l} q$, if 
- whenever $p \stackrel{a(x)}{\longrightarrow} p^{\prime}$ with $x \notin f n(p, q)$ then $q \stackrel{a(x)}{\Longrightarrow} q^{\prime \prime}$ for some $q^{\prime \prime}$ s.t for any $y$ there is $q^{\prime \prime}[y / x] \stackrel{\hat{\tau}^{\prime}}{\Longrightarrow} q^{\prime}$ and $p^{\prime}[y / x] \approx_{l} q^{\prime}$.

- whenever $p \stackrel{\alpha}{\longrightarrow} p^{\prime}$ for any other action $\alpha$ with $\operatorname{bn}(\alpha) \cap f n(p, q)=\emptyset$ then $q \stackrel{\alpha}{\Longrightarrow} q^{\prime}$ for some $q^{\prime}$ and $p^{\prime} \approx_{l} q^{\prime}$.

And similarly for $u$.

$t$ is late observation congruent to $u$, written $t \simeq_{l} u$, if $t \sigma \simeq_{l} u \sigma$ for every substitution $\sigma$.

The late symbolic double arrows are defined as the least relations satisfying the following rules:

- $t \stackrel{\text { true, }, E}{\Longrightarrow} t$.

- $t \stackrel{M}{\longrightarrow} \alpha_{L} u$ implies $t \stackrel{M_{\alpha}^{\alpha}}{\longrightarrow} u$.

- $t \stackrel{M}{\longrightarrow} \tau_{L}^{\tau} \stackrel{N, \alpha}{\Longrightarrow} u$ implies $t \stackrel{M N, \alpha}{\Longrightarrow} u$.

- If $\alpha$ does not have the form $a(x)$ then $t \stackrel{M_{,} \alpha}{\Longrightarrow} \stackrel{N, \tau}{\longrightarrow} L$ implies $t \stackrel{M N, \alpha}{\Longrightarrow} u$.

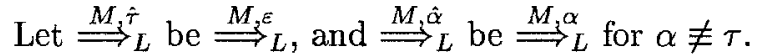

Definition 2.6 A condition indexed family of symmetric relations $\mathcal{S}=\left\{S^{C}\right\}$ is a late weak symbolic bisimulation if $(t, u) \in S^{C}$ implies whenever $t \stackrel{M, \alpha}{\longrightarrow} t^{\prime}$ with $b n(\alpha) \cap f n(t, u)=\emptyset$, then for each $D \in M C E_{f n(t, u)}(C \cup M)$ there is a $u \stackrel{N, \hat{\beta}_{2}}{\Longrightarrow} u^{\prime}$ such that $D \Rightarrow N, \alpha=^{D} \beta$, and

- If $\alpha \equiv a(x)$ then for each $D^{\prime} \in M C E_{f n(t, u) \cup\{x\}}(D)$ there is $u^{\prime}{\stackrel{N^{\prime}}{\Longrightarrow}}_{L}^{\hat{\tau}} u^{\prime \prime}$ s.t. $D^{\prime} \Rightarrow N^{\prime}$ and $\left(t^{\prime}, u^{\prime \prime}\right) \in S^{D^{\prime}}$.

- If $\alpha \equiv \bar{a}(x)$ then $\left(t^{\prime}, u^{\prime}\right) \in S^{D \cup\left\{x \neq y \mid y \in f n\left(\alpha . t^{\prime}, \beta \cdot u^{\prime}\right)\right\}}$.

- Otherwise $\left(t^{\prime}, u^{\prime}\right) \in S^{D}$.

Let $\approx_{L}$ be the largest late weak symbolic bisimulation.

Proposition $2.7 t \approx_{L}^{C} u$ iff $t \sigma \dot{\sim}_{l} u \sigma$ for every $\sigma \models C$.

Late symbolic observation equivalence is defined in terms of $\approx_{L}$ as usual:

Definition 2.8 Two terms $t, u$ are late symbolic observation equivalent over $C$, written $t \simeq_{L}^{C} u$, if whenever $t \stackrel{M, \alpha}{\longrightarrow} t^{\prime}$ with $b n(\alpha) \cap f n(t, u)=\emptyset$, then for each $D \in M C E_{f n(t, u)}(C \cup M)$ there is a $u \stackrel{N, \beta}{\Longrightarrow} u^{\prime}$ such that $D \Rightarrow N, \alpha={ }^{D} \beta$, and

- If $\alpha \equiv a(x)$ then for each $D^{\prime} \in M C E_{f n(t, u) \cup\{x\}}(D)$ there is $u^{\prime} \stackrel{M^{\prime}, \hat{T}}{\Longrightarrow}{ }_{L}^{\prime \prime} u^{\prime \prime}$ s.t. $D^{\prime} \Rightarrow M^{\prime}$ and $t^{\prime} \approx_{L}^{D^{\prime}} u^{\prime \prime}$. 


$$
\begin{aligned}
& \text { ALPHA } \frac{}{\text { true } \triangleright t=u} t \equiv u \\
& \text { AXIOM } \frac{}{\text { true } \triangleright t=u} t=u \text { an axiom instance } \\
& \text { CHOICE } \frac{C \triangleright t_{i}=u_{i}}{C \triangleright t_{1}+t_{2}=u_{1}+u_{2}} \quad \text { TAU } \frac{C \triangleright t=u}{C \triangleright \tau . t=\tau . u} \\
& \text { L-INPUT } \frac{C \triangleright t=u}{C \triangleright a(x) \cdot t=b(x) \cdot u} C \Rightarrow a=b, x \notin n(C) \\
& \text { OUTPUT } \frac{C \triangleright t=u}{C \triangleright \bar{a} x \cdot t=\bar{b} y \cdot u} C \Rightarrow a=b, C \Rightarrow x=y \\
& \text { MATCH } \frac{C \cup\{x=y\} \triangleright t=u \quad C \cup\{x \neq y\} \triangleright \mathbf{0}=u}{C \triangleright[x=y] t=u} \\
& \operatorname{RES} \frac{C \cup\{x \neq y \mid y \in f n((x) t,(x) u)\} \triangleright t=u}{C \triangleright(x) t=(x) u} x \notin n(C) \\
& \operatorname{CUT} \frac{C \cup\{x=y\} \triangleright t=u \quad C \cup\{x \neq y\} \triangleright t=u}{C \triangleright t=u} \\
& \text { CONSEQ } \frac{C \triangleright t=u}{C^{\prime} \triangleright t=u} C^{\prime} \Rightarrow C \quad \text { ABSURD } \frac{}{\text { false } \triangleright t=u}
\end{aligned}
$$

Figure 3: The Inference Rules for Late Symbolic Bisimulation

- If $\alpha \equiv \bar{a}(x)$ then $t^{\prime} \approx_{L}^{D \cup\left\{x \neq y \mid y \in f n\left(\alpha \cdot t^{\prime}, \beta \cdot u^{\prime}\right)\right\}} u^{\prime}$.

- Otherwise $t^{\prime} \approx_{L}^{D} u^{\prime}$.

And similarly for $u$.

Proposition $2.9 t \simeq_{L}^{C} u$ iff $t \sigma \simeq_{l} u \sigma$ for every $\sigma \models C$.

\section{The Inference System}

In this section we will concentrate on a sublanguage without the parallel composition operator |. Later we will see that this operator can be axiomatised easily.

The inference system for late symbolic bisimulation is reported in Figure 3. The judgements are conditional equations of the form

$$
C \triangleright t=u
$$

where $t=u$ is an equation in the $\pi$-calculus and $C$ is a condition. 


$\begin{array}{llll}\text { S1 } & X+\mathbf{0}=X & \mathrm{R} 1 & (x) 0=0 \\ \text { S2 } & X+X=X & \mathrm{R} 2 & (x) \alpha \cdot X=\alpha .(x) X \text { if } x \notin n(\alpha) \\ \text { S3 } & X+Y=Y+X & \mathrm{R} 3 & (x) \alpha . X=0 \text { if } x \text { is the port of } \alpha \\ \text { S4 } & (X+Y)+Z=X+(Y+Z) & \mathrm{R} 4 & (x)(y) X=(y)(x) X \\ & & \mathrm{R} 5 & (x)(X+Y)=(x) X+(x) Y \\ \text { T1 } & \alpha . \tau . X=\alpha . X & \mathrm{~T} 2 & X+\tau . X=\tau . X \\ \mathrm{~T} 3 & \alpha .(X+\tau . Y)+\alpha . Y=\alpha .(X+\tau . Y)\end{array}$

Figure 4: The Axioms for Choice, Restriction and $\tau$

In order to derive interesting equations from this inference system we need some standard axioms. These include the axioms for the choice operator (S1 - S4), the axioms for the restriction operator (R1 - R5), and the $\tau$-laws (T1 - T3) in Figure 4. Let $\mathcal{A}_{s}=\{S 1, S 2, S 3, S 4, R 1, R 2, R 3, R 4, R 5\}$ and $\mathcal{A}_{w}=$ $\mathcal{A}_{s} \cup\{T 1, T 2, T 3\}$.

We write $\vdash C \triangleright t=u$ and $\vdash_{w L} C \triangleright t=u$ to mean that $C \triangleright t=u$ can be derived from $\mathcal{A}_{s}$ and $\mathcal{A}_{w}$, respectively, using the above inference rules. It is immediate from the definition that $\vdash C \triangleright t=u$ implies $\vdash_{w L} C \triangleright t=u$. Some properties of these proof systems are listed bellow.

Proposition 3.1 1. If $C \Rightarrow M$ and $\vdash C \triangleright t=u$ then $\vdash C \triangleright M t=u$.

2. If $C \cup M \Rightarrow$ false then $\vdash C \triangleright M t=\mathbf{0}$.

3. $\vdash t=t+M t$.

Proposition 3.2 If $\vdash D \triangleright t=u$ for each $D \in M C E_{V}(C)$ then $\vdash C \triangleright t=u$.

Proposition 3.3 1. $\vdash(x)[x=x] t=(x) t$.

2. $\vdash(x)[x=y] t=0$.

3. $\vdash(x)[y=z] t=[y=z](x) t$.

The soundness and completeness of $\vdash$ w.r.t $\sim_{L}$ (hence $\sim_{l}$ ) have been proved in [Lin94b]. The rest of this section is devoted to the proof of completeness result for $\vdash_{w L}$.

If $a \neq x$ then we abbreviate $(x) \bar{a} x . t$ as $\bar{a}(x) . t . \bar{a}(x)$ is a derived action called bound output. Using RES the following two equations can be easily derived from $\mathrm{T} 1$ and T3:

$$
\begin{gathered}
\bar{a}(x) \cdot \tau \cdot X=\bar{a}(x) \cdot X \\
\bar{a}(x) \cdot(X+\tau \cdot Y)+\bar{a}(x) \cdot Y=\bar{a}(x) \cdot(X+\tau \cdot Y)
\end{gathered}
$$

So from now on we will allow the action $\alpha$ in $\mathrm{T} 1$ and $\mathrm{T} 3$ to range over bound output as well.

We also have the following derived inference rule for bound output: 
Proposition 3.4 Suppose $C \Rightarrow a=b, x \notin n(C)$. If $\vdash C \cup\{x \neq y \mid y \in$ $f n(\bar{a}(x) \cdot t, \bar{b}(x) \cdot u)\} \triangleright t=u$ then $\vdash C \triangleright \bar{a}(x) \cdot t=\bar{b}(x) \cdot u$.

A term is restriction-free if, using the above abbreviation, it does not explicitly contain any occurrences of the restriction operator.

A restriction-free term is a standard form if it has the form $\sum_{i} M_{i} \alpha_{i} \cdot t_{i}$ and each $t_{i}$ is a standard form. A standard form $\sum_{i} M_{i} \alpha_{i} . t_{i}$ is a full standard form if

- $t \stackrel{M_{\alpha}^{\alpha}}{\longrightarrow} t^{\prime}$, where $b n(\alpha) \cap n(M)=\emptyset$, implies $t \stackrel{M, \alpha}{\longrightarrow} t^{\prime} ;$

- Each $t_{i}$ is in full standard form.

The following two crucial lemmas hold:

Lemma 3.5 (Absorption) If $t \stackrel{M_{\alpha} \alpha}{\Longrightarrow} t^{\prime}$ with $b n(\alpha) \cap n(M)=\emptyset$ then $\vdash_{w L} t=$ $t+M \alpha \cdot t^{\prime}$.

Lemma 3.6 If $t \stackrel{M_{\tilde{r}}}{\Longrightarrow}{ }_{L}^{\prime}, C \Rightarrow M$ and $\vdash_{w L} C \triangleright \tau . t^{\prime}=\tau$.u, then $\vdash_{w L} C \triangleright \tau . t=$ $\tau . t+\tau . u$.

Let the height of a term $t,|t|$, be defined as the maximum depth of action nesting in $t$.

Lemma 3.7 For any term $t$ there is a full standard form $t^{\prime}$ such that $\vdash_{w L} t=t^{\prime}$ and $\left|t^{\prime}\right| \leq|t|$.

The following proposition relates observation equivalence to weak bisimulation. It will play an important rôle in the proof of the completeness theorem.

Proposition 3.8 Suppose $D \in M C_{f n(t, u)}$. Then $t \approx_{L}^{D} u$ if and only if either $t \simeq_{L}^{D} u$, or $\tau . t \simeq_{L}^{D} u$, or $t \simeq_{L}^{D} \tau . u$.

Using Lemmas 3.7, 3.6 and 3.8 we can prove the main theorem of this paper:

Theorem 3.9 (Completeness of $\vdash_{w L}$ ) If $t \simeq_{L}^{C} u$ then $\vdash_{w L} C \triangleright t=u$.

Proof: By Lemma 3.7 we may assume $t, u$ are standard forms: $t \equiv \sum_{i} M_{i} \alpha_{i} \cdot t_{i}$, $u \equiv \sum_{j} N_{j} \beta_{j} . u_{j}$. We may further assume that bound actions in $\alpha_{i}, \beta_{j}$ use the same bound name $z \notin f n(t, u, C)$. The proof is by induction on the joint height of $t$ and $u$. By Proposition 3.2 we need only to show $\vdash_{u L} D \triangleright t=u$ for each $D \in$ $M C E_{f n(t, u, C)}(C)$, and for this it is sufficient to show $t_{w L} D \triangleright t=t+N_{j} \beta_{j} . u_{j}$ for each $j$.

If $D \nRightarrow N_{j}$ then, since $D$ is maximally consistent, $D \cup N_{j} \Rightarrow$ false. So by Proposition 3.1, $\vdash_{w L} D \triangleright N_{j} \beta_{j} \cdot u_{j}=0$. Hence $\vdash_{w L} D \triangleright t=t+N_{j} \beta_{j} . u_{j}$ by S1.

Now assume $D \Rightarrow N_{j}$ (then $D \in M C E_{f n(t, u, C)}\left(C \cup N_{j}\right)$ ), and consider four cases according to the type of $\beta_{j}$. 
- $\beta_{j} \equiv \tau$. Then $u \stackrel{N_{j}, \tau}{\longrightarrow} u_{j}$. Since $t \simeq_{L}^{C} u$ and $t$ is a full normal from, $t \stackrel{M_{i}, \tau}{\longrightarrow} t_{i}$ for some $i$ s.t. $D \Rightarrow M_{i}$ and $t_{i} \approx_{L}^{D} u_{j}$. By Proposition 3.8 we have either $t_{i} \simeq_{L}^{D} u_{j}$, or $\tau \cdot t_{i} \simeq_{L}^{D} u_{j}$, or $t_{i} \simeq_{L}^{D} \tau . u_{j}$. In the first case we can apply induction to get $\vdash_{w L} D \triangleright t_{i}=u_{j}$, and by TAU, $\vdash_{w L} D \triangleright \tau \cdot t_{i}=\tau \cdot u_{j}$. In the second case, by Lemma 3.7 there is a full normal form $t^{t}$ with height no greater than $t$ s.t. $\vdash_{w L} \tau . t_{i}=t^{\prime}$. By the soundness of $\vdash_{w L}, t^{\prime} \simeq_{L}^{t r u e} \tau \cdot t_{i}$, hence $t^{\prime} \simeq_{L}^{D} u_{j}$. By induction $\vdash_{w L} D \triangleright t^{\prime}=u_{j}$, so $\vdash_{w L} D \triangleright \tau \cdot t_{i}=u_{j}$. Apply TAU and T1 we get $\vdash_{w L} D \triangleright \tau . t_{i}=\tau \cdot u_{j}$. In the third case we can similarly get $\vdash_{w i} D \triangleright \tau \cdot t_{i}=\tau \cdot u_{j}$. Since $D \Rightarrow M_{i}$ and $D \Rightarrow N_{j}$, by Proposition $3.1 \vdash_{w L} D \triangleright M_{i} \tau \cdot t_{i}=N_{j} \tau \cdot u_{j}$. Finally by $\mathrm{S} 2, \vdash_{w L} D \triangleright t=$ $t+N_{j} \tau \cdot u_{j}$.

- $\beta_{j} \equiv \bar{b}(z)$. Then $u \stackrel{N_{j}, \bar{b}(z)}{\longrightarrow} u_{j}$, so $t \stackrel{M_{1}, \bar{a}_{2}(z)}{\longrightarrow} t_{L} \stackrel{M^{\prime}, \hat{\tau}}{\longrightarrow} t^{\prime}$ for some $i$ s.t.

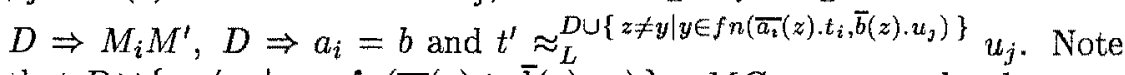
that $D \cup\left\{z \neq y \mid y \in f n\left(\overline{a_{i}}(z) \cdot t_{i}, \bar{b}(z) \cdot u_{j}\right)\right\} \in M C_{f n\left(t_{i}, u_{j}\right)}$, so by the same argument as in the $\tau$ case, $\vdash_{w L} D \cup\left\{z \neq y \mid y \in f n\left(\overline{a_{i}}(z) . t_{i}, \bar{b}(z) \cdot u_{j}\right)\right\} \triangleright$ $\tau . t^{\prime}=\tau . u_{j}$. By Lemma 3.6, $\vdash_{w L} D^{\prime} \triangleright \tau . t_{i}=\tau . t_{i}+\tau . u_{j}$. By Proposition 3.4, $\vdash_{w L} D \triangleright \overline{a_{i}}(z) \cdot \tau \cdot t_{i}=\overline{a_{i}}(z) \cdot\left(\tau \cdot t_{i}+\tau \cdot u_{j}\right)$. Hence

$$
\begin{aligned}
\vdash_{w L} D \triangleright \overline{a_{i}}(z) \cdot t_{i} & \stackrel{T 1}{=} \overline{a_{i}}(z) \cdot \tau \cdot t_{i} \\
& =\overline{a_{i}}(z) \cdot\left(\tau \cdot t_{i}+\tau \cdot u_{j}\right) \\
& \stackrel{T 3}{=} \overline{a_{i}}(z) \cdot\left(\tau \cdot t_{i}+\tau \cdot u_{j}\right)+\overline{a_{i}}(z) \cdot u_{j} \\
& =\overline{a_{i}}(z) \cdot t_{i}+\overline{a_{i}}(z) \cdot u_{j} \\
& =\overline{a_{\imath}}(z) \cdot t_{i}+\bar{b}(z) \cdot u_{j}
\end{aligned}
$$

Since $D \Rightarrow M_{i}$ and $D \Rightarrow N_{j}$, by Proposition 3.1, $\vdash_{w L} D \triangleright M_{i} \overline{a_{i}}(z) \cdot t_{i}=$ $M_{i} \overline{a_{i}}(z) \cdot t_{i}+N_{j} \bar{b}(z) \cdot u_{j}$. Hence $\vdash_{w L} D \triangleright t=t+N_{j} \bar{b}(z) \cdot u_{j}$ by $\mathrm{S} 2$.

- The other two cases are similar.

\section{The Early Case}

Early weak (concrete) bisimulation and congruence have been discussed in [Wa194], here we only give the definitions for thier symbolic counterpart.

To define early symbolic double arrow, $\Longrightarrow_{E}$, we simply remove the "noninput action" restriction to the last rule in the definition of late double arrow in Section 2.3 , with the other rules unchanged.

Definition 4.1 A condition indexed family of symmetric relations $\mathcal{S}=\left\{S^{C}\right\}$ is an early weak symbolic bisimulation if $(t, u) \in S^{C}$ implies

whenever $t \stackrel{M, \alpha}{\longrightarrow} t^{\prime}$ with $b n(\alpha) \cap f n(t, u)=\emptyset$, then for each $D \in$ $M C E_{V}(C \cup M)$ there is a $u \stackrel{N, \hat{\beta}}{\Longrightarrow} u^{\prime}$ such that $D \Rightarrow N, \alpha==^{D} \beta$, 
and $\left(t^{\prime}, u^{\prime}\right) \in S^{D^{\prime}}$, where

$$
\begin{gathered}
V= \begin{cases}f n(t, u) \cup\{x\} & \text { if } \alpha \equiv a(x) \\
f n(t, u) & \text { otherwise }\end{cases} \\
D^{\prime}= \begin{cases}D \cup\left\{x \neq y \mid y \in f n\left(\alpha . t^{\prime}, \beta . u^{\prime}\right)\right\} & \text { if } \alpha \equiv \bar{a}(x) \\
D & \text { otherwise }\end{cases}
\end{gathered}
$$

Let $\approx_{E}$ be the largest early weak symbolic bisimulation.

Early symbolic observation equivalence $\simeq_{E}$ can then be defined in terms of $\approx_{E}$ as usual.

Proposition $4.2 t \simeq_{E}^{C} u$ iff $t \sigma \simeq_{e} u \sigma$ for every $\sigma \vDash C$.

The proof systems for early weak equivalence can be obtained by replacing the L-INPUT rule in Figure 3 with the following one

$$
\text { E-INPUT } \frac{C \triangleright \sum_{i \in I} \tau \cdot t_{i}=\sum_{j \in J} \tau \cdot u_{j}}{C \triangleright \sum_{i \in I} a_{i}(x) \cdot t_{i}=\sum_{j \in J} b_{j}(x) \cdot u_{j}} \quad \begin{aligned}
& C \Rightarrow a_{i}=b_{j}, i \in I, j \in J(C) \\
& x \notin n(C)
\end{aligned}
$$

Let us write $\vdash_{w E} C \triangleright t=u$ to mean $C \triangleright t=u$ can be derived from this new proof system. It is easy to see that, in the presence of the TAU rule, L-INPUT can be derived from E-INPUT, but not the other way round. So $\vdash_{w E}$ is strictly stronger than $\vdash_{w L}$.

All the lemmas in the previous section hold for $\vdash_{w E}$ as well. And we have the following counterpart of Theorem 3.9:

Theorem 4.3 (Completeness of $\vdash_{w E}$ ) If $t \simeq_{E}^{C} u$ then $\vdash_{w E} C \triangleright t=u$.

\section{Other Operators}

\subsection{Parallel Composition}

To deal with parallel composition all we need is a suitable form of expansion law which is presented in Figure 5. With this law it is standard that any term containing parallel operator can be reduced to a term without it, hence the normal form lemma still holds, as does the completeness theorem.

\subsection{Mismatch}

To include mismatch, i.e. testing inequality between names, into the language we first extend the operational semantics by including the following two rules: "mismatch" and "Mismatch" in Figure 1 and Figure 2, respectively (now $M$ ranges over conditions)

$\operatorname{mismatch} \frac{t \stackrel{\alpha}{\longrightarrow} t^{\prime}}{[x \neq y] t \stackrel{\alpha}{\longrightarrow} t^{\prime}} x \neq y$

$$
\text { Mismatch } \frac{t \stackrel{M, \alpha}{\longrightarrow} t^{\prime}}{[x \neq y] t \stackrel{M[x \neq y], \alpha}{\longrightarrow} t^{\prime}}
$$


Let $t \equiv \sum_{i} M_{i} \alpha_{i} \cdot t_{i}$ and $u \equiv \sum_{j} N_{j} \beta_{j} \cdot u_{j}$ with $b n\left(\alpha_{i}\right) \cap f n(u)=b n\left(\beta_{j}\right) \cap f n(t)=$ $\emptyset$. Then

$$
t \mid u=\sum_{i} M_{i} \alpha_{i} \cdot\left(t_{i} \mid u\right)+\sum_{j} N_{j} \beta_{\jmath} \cdot\left(t \mid u_{j}\right)+\sum_{\alpha_{2}} M_{o p p \beta_{j}} N_{j}\left[a_{i}=b_{j}\right] \tau \cdot v_{\imath \jmath}
$$

where $\alpha_{i}$ opp $\beta_{j}$ and $v_{i j}$ are defined as follows

1. $\alpha_{i} \equiv a(x), \beta_{j} \equiv \bar{b} y$; then $v_{i j} \equiv t_{i}[y / x] \mid u_{j}$;

2. The converse of the above clause;

3. $\alpha_{i} \equiv a(x), \beta_{j} \equiv \bar{b}(y)$; then $v_{i j} \equiv(z)\left(t_{i}[z / x] \mid u_{j}[z / y]\right)$ with $z \notin f n(t, u)$;

4. The converse of the above clause.

Figure 5: The Expansion Law

The inference rule for mismatch is dual to that for match:

$$
\text { MISMATCH } \frac{C \cup\{x \neq y\} \triangleright t=u \quad C \cup\{x=y\} \triangleright 0=u}{C \triangleright[x \neq y] t=u}
$$

With this rule Proposition 3.1 can be generalised to allow $M$ to be an arbitrary condition, not just a match.

Now, in a normal form $\sum_{i} M_{i} \alpha_{i} \cdot t_{i}, M_{i}$ may contain inequality tests as well as equality tests. This does not affect the proofs of the normal form lemma and the completeness theorem.

\section{Conclusions And Related Work}

We have presented complete proof systems for late and early weak observation equivalences for the $\pi$-calculus. The inference system for the early equivalence is obtained by adding the E-INPUT rule to the inference system for the late equivalence. To enrich the language with the mismatch construction we only need to extend the inference systems with a single rule, namely MISMATCH. In each case the proof system for weak equivalence is obtained by adding the standard $\tau$-laws to the one for the strong equivalence. Thus in all we have eight proof systems. The relationship between them are pictured in Figure 6, where arrows stand for "extension" and $\pi^{\neq}$is the version of $\pi$-calculus enriched with mismatch.

Our results verify a conjecture posed in [MPW92, PS93, BD94]: the three $\tau$-laws are sufficient for the transition from strong to weak bisimulation equivalences for the $\pi$-calculus, as in the case of $C C S$.

The current work inherits the main ideas from our previous work on general message-passing process calculi [HL92, HL93]. As the process calculi considered 


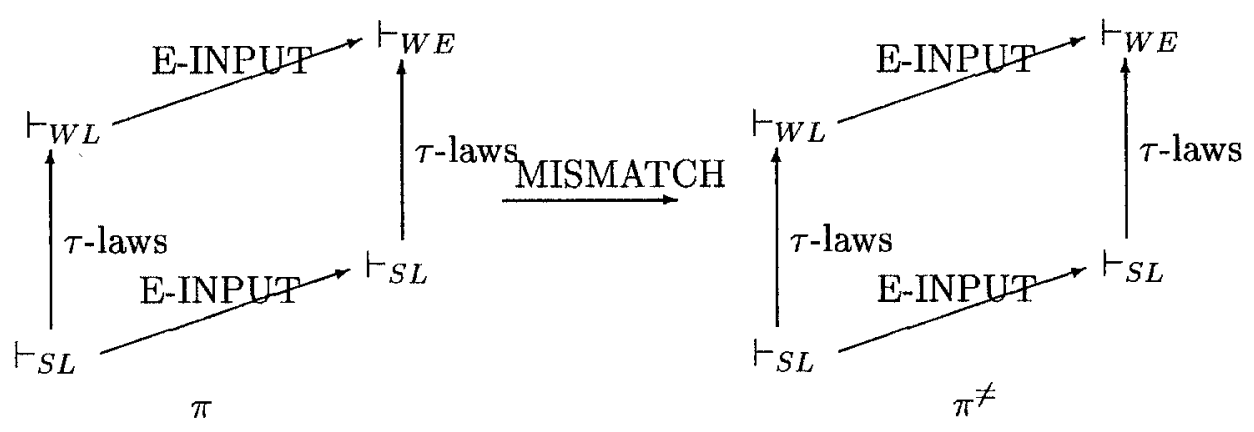

Figure 6: The Relationship between Proof Systems

there take the languages for data and boolean expressions as parameters, the completeness results in those papers are relative to data reasoning. Here, by exploiting the specific features presented in the $\pi$-calculus, there is no need to refer to an "oracle" for the data domain and the completeness results are no longer relative to reasoning about data.

Parrow and Sangiorgi ([PS93]) provided complete equational theories for both late and early strong congruences for name-passing calculi, i.e. extensions of the $\pi$-calculus with the mismatch construction. Mismatch plays an essential rôle in at least two places: the definition of normal form and the axiom for early equivalence. The equational characterisation of the restriction operator also relies on mismatch.

In [BD94] Moreale and De Nicola proposed "symbolic" semantics and proof systems very similar to [Lin94b]. Again they work with the extension of the $\pi$ calculus with mismatch instead of the $\pi$-calculus proper, and only strong equivalences were treated.

[Liu94] gives a symbolic version of distinction-indexed bisimulation for a sublanguage of the $\pi$-calculus (without the match operator) and shows that this notion of bisimulation captures the conventional bisimulation equivalences. But no proof system is considered there.

In [San93] another notion of bisimulation, called open bisimulation, for the $\pi$-calculus is proposed, along with an equational axiomatisation. A "symbolic" characterisation of open bisimulation is also given. To deal with the restriction operator, distinctions have to be exploited as indices in the definition of open bisimulation as well as in the axiomatisation. Open bisimulation has been further studied in [Wal94].

We are not aware any other published work on axiomatising weak bisimulation equivalences in the $\pi$-calculus.

Acknowledgment Thanks to Matthew Hennessy for reading a draft of this paper and suggesting many improvements. 


\section{References}

[BD92] M. Boreale and R. DeNicola. Testing equivalence for mobile processes. In CONCUR'92, number 630 in Lecture Notes in Computer Science, pages 2 - 16. Springer-Verlag, 1992.

[BD94] M. Boreale and R. DeNicola. A symbolic semantics for the $\pi$ calculus. In CONCUR'94, Lecture Notes in Computer Science. Springer-Verlag, 1994.

[Hen91] M. Hennessy. A model for the $\pi$-calculus. Technical Report 8/91, CSAI, University of Sussex, 1991.

[HL92] M. Hennessy and H. Lin. Symbolic bisimulations. Technical Report 1/92, CSAI, University of Sussex, 1992. to appear in Theoretical Computer Science.

[HL93] M. Hennessy and H. Lin. Proof systems for message-passing process algebras. In CONCUR'93, number 715 in Lecture Notes in Computer Science, pages 202-216, 1993.

[Lin94a] H. Lin. Complete inference systems for weak bisimulation equivalences in the $\pi$-calculus. Report ISCAS-LCS-94-11, Laboratory for Computer Science, Institute of Software, Chinese Academy of Science, 1994.

[Lin94b] H. Lin. Symbolic bisimulations and proof systems for the $\pi$-calculus. Report 7/94, Computer Science, University of Sussex, 1994.

[Liu94] X. Liu. Characterizing bisimulation congruence in the $\pi$-calculus. In CONCUR'94, Lecture Notes in Computer Science. Springer-Verlag, 1994.

[Mil89] R. Milner. Communication and Concurrency. Prentice-Hall, 1989.

[MPW92] R. Milner, J. Parrow, and D. Walker. A calculus of mobile proceses, part I,II. Information and Computation, 100:1-77, 1992.

[PS93] J. Parrow and D. Sangiorgi. Algebraic theories for name-passing calculi. Report ECS-LFCS-93-262, LFCS, University of Edinburgh, 1993.

[San93] D. Sangiorgi. A theory of bisimulation for the $\pi$-calculus. In $C O N$ CUR'93, number 715 in Lecture Notes in Computer Science, 1993.

[Wal94] D. Walker. On bisimulation in the $\pi$-calculus. In CONCUR'94, Lecture Notes in Computer Science. Springer-Verlag, 1994. 